\title{
Compression of Noisy Images based on Sparsification using Discrete Rajan Transform
}

\author{
Kethepalli Mallikarjuna \\ Research Scholar \\ JNTUK University, Kakinada \\ Andhra Pradesh, India
}

\author{
Kodati Satya Prasad \\ Professor, Dept. of ECE \\ JNTUKU Engineering College \\ Kakinada, A.P, India
}

\author{
M.V. Subramanyam \\ Principal, SREC, \\ Nandyal, Kurnool, \\ Andhra Pradesh, India
}

\begin{abstract}
Image compression is usually carried out to reduce the amount of data required to store or communicate a digital image or video. The basic idea involved in the reduction process is removal of redundant data. Image compression exploits the fact that all images are not equally likely. In this regard a good number of Compression algorithms have been developed by researchers. As an alternative to the available traditional approaches, this paper presents the use of Discrete Rajan Transform for sparsification and image compression of noisy images. Discrete Rajan Transform is effective in introducing sparsity in images and thereby improving compressibility, the compromise being acceptable loss of data. In this paper, images with Gaussian, Poisson, Salt and pepper, and speckle noise have been investigated using the proposed method and a brief analysis is carried out in terms of perception of images as well in terms of three important parameters, Peak Signal-to-Noise Ratio, Mean Squared Error and Compression Ratio. On simulation, it was observed that DRT yielded higher quality image than the other candidate transforms used, namely Discrete Cosine Transform and Discrete Wavelet Transform.
\end{abstract}

\section{General Terms}

Compression ratio, Image compression, Mean Squared Error, Peak Signal-to-Noise Ratio, Quality of reconstructed image.

\section{Keywords}

Compression, Discrete Rajan Transform, Noisy Images, Sparsification.

\section{INTRODUCTION}

Regular developments of techniques and methodologies in the field of imaging and multimedia have invariably caused visual information contained in images to act as one of the main sources for knowledge acquisition. It is inevitable to confront unwanted noise and artifacts in images and video slides while acquiring, processing, transmitting and storing visual information because of which there are degradation in the visual quality of images and video slices. This has led to an increase in the requirement of digital images and multimedia information transmission [1]. Images contain large amounts of redundant information and in order to improve transmission process, compression techniques are being used. Compression is essential to ease transmission of images and other multimedia information [2-5]. In the past few decades, the cost involved in storage has been reduced. However, considering the large amount data storage and transmission requirements, the pace of development is still far from our expectations [6]. However, quite a number of techniques and methodologies have been developed and being developed to have maximum information content in various data with minimum storage possibilities. It is usually desired to go in for lossless data compression which is unlikely for data with large entropy. On the other hand, lossy compression could be an alternative solution but with a compromise on the trade-off to achieve greater compression ratios. Lossy compression techniques do play a significant role in signal and image processing. The Joint Photographic Experts Group (JPEG) standardized the notion of transforming spatial domain images to frequency domain spectra for filtering unwanted information so that better data compression is achieved [7-8]. Though complexity of computation has always been a bottleneck in data compression, present computing techniques have indeed shifted the demand away from speed but towards storage capacity limitations. In fact, storage cost involved in archiving digital image data has become the point of concern to research and development pertaining to reliable and efficient data reduction techniques which would ensure optimal information recovery. As on date, one can observe that the ever increasing demand for high resolution images of good visual quality in terms of color and visual information keeps the research tempo on especially in the area of image compression [9]. As a result many image compression and coding techniques are being developed which work on either spatial domain or spectral domain [10-13]. Transform domain fast algorithms do offer good data compression, but at the cost of considerable processing time-[14]. It is also a well known fact that unwarranted introduction of noise patterns due to these algorithms degrade their performance. Degradation of images can be witnessed even before the encoding stage. For example, in the case of transmission of tomography images, they are affected by noise which is data-dependent and can be modeled as Poisson noise [15-16]. Generally, images formed at low light levels are also corrupted by this kind of noise [17]. Similarly, there are other types of noises that affect the quality of images such as Gaussian noise, salt and pepper noise and Speckle Noise. Image compression can give rise to artifacts and distortions and this makes the output image lose some amount of data and also disagreeable. Most images are visualized and hence the characterization of compressed image quality has to be done considering the Human Visual System (HVS) [18-19]. Widely used quantitative parameters for design and comparison of various techniques used in compression of images are Mean Square Error (MSE) and Peak Signal-to-Noise Ratio (PSNR). However, some research shows that these quantitative criteria are not adequate / sufficient to describe the quality of the compression technique [18-23]. The standard JPEG format was analyzed and changes made into the new JPEG2000 [24- 25]. Hence, for the purpose of data compression, different calculation methods are being used. These methods are broadly classified into two categories based on the distortions in the input image lossless compression and lossy compression. Lossless compression, as the name implies, is the technique that involves no loss of data during the compression process and the pre-compressed signal can be restored. Contrary to the Lossless compression technique, Lossy compression technique involves loss of data [26]. However, it has to be understood that images are complex data sets and 
unfortunately there is hardly a transform domain technique found in literature that can optimally and efficiently represent an image without causing severe information loss. For images with oscillatory textures, Fourier transform would be a better choice for an effective sparse representation. Alternatively, use of DWT would ensure better performance for images with isolated singular textures [27]. Apart from these traditional approaches, one could explore the possibilities of using DRT for image sparsification and data compression and this paper is all about discussing such a possibility. DRT was also tried on noisy images (Poisson noise, Gaussian noise, salt and pepper noise and Speckle Noise) and MSE and PSNR evaluated and results reported in this paper.

\section{DISCRETE RAJAN TRANSFORM}

Rajan Transform (RT) is a constructive analogue of Hadamard Transform but a homomorphic map exhibiting permutation invariance property. Various pattern recognition algorithms like thinning, edge detection, contour detection, detection of curves and lines and isolation of certain points in digital images have been effectively carried out by RT. Basically Rajan Transform is a fast algorithm which has the operational semantics of Decimation-In-Frequency (DIF) Fast Fourier Transform algorithm [28]. Discrete Rajan Transform (DRT) is the generalized form of Rajan Transform, which exhibits the set theoretic property of isomorphism. This is due to the fact that the auxiliary phasor information associated with the DRT spectrum of a signal is known apriori. Similar to Fast Fourier Transform, Discrete Rajan Transform is a fast algorithm whose details could be found elsewhere [29].

\subsection{Image Sparsification using DRT}

A finite $\mathrm{N}$ dimensional vector is viewed as a sparse signal if majority of its sample values are zero or almost zero. If $\mathrm{K}$ of the $\mathrm{N}$ sample values of a signal are found to be nonzero and the remaining $(\mathrm{N}-\mathrm{K})$ are zero, then the signal is termed as $\mathrm{K}$ sparse signal. A signal is sparsified by making those sample values which are less than a threshold value to zero. Normally every image transform provides certain amount of sparsification. As a result of an analytical study, DRT has been found to provide better sparsification than Discrete Cosine Transform (DCT) and Discrete Wavelet Transform (DWT). Image sparsification could possibly lead to improved image compression. Sparsification based image compression using DRT provides better Peak Signal-to-Noise Ratio than that provided using DCT and DWT [29-31].

\subsection{Noisy Image Compression using DRT}

In order to improve sparse representation, a special case of DRT is used where the auxiliary phasor information obtained at every stage of computation is considered as 1 . The block diagram for compression of noisy images using DRT sparsifying transform is shown in the Figure 1. After block sampling from the given noisy image, the forward DRT is applied on the block of size 1X8. Among the eight spectral coefficients the first and the fifth coefficients carries maximum information. Hence the first and fifth coefficients are retained and all other six coefficients are further sparsified to zero. This process is repeated for all blocks until the entire image is scanned. Then run length encoding is used to obtain compressed image. The compression ratio has been calculated from the compressed image and original image. The compression ratio is defined as the ratio of the number of bits required to represent the original image to that required to represent the compressed image i.e.,

$C R=\frac{\text { number of bits required to represent the original image }}{\text { number of bits required to represent the compressed image }}$

On the receiving side, after applying run length decoding and inverse DRT, an appropriate filter was used to get back the approximated reconstructed image. The performance parameters MSE and PSNR were calculated from the filtered reconstructed and original images using the formulae:

$$
\begin{gathered}
M S E=\frac{1}{M N} \sum_{x=1}^{M} \sum_{y=1}^{N}\left[I(x, y)-I^{\prime}(x, y)\right]^{2} \\
P S N R=10 \log _{10}\left(\frac{255^{2}}{M S E}\right)
\end{gathered}
$$

The process is repeated using DCT and DWT. This entire procedure is applied for images with Gaussian noise, Poisson noise, Salt \& Pepper noise, and Speckle noise. This algorithm can be summarized as follows:

1) Input an image of Dimension (W X W)

2) Add noise (Gaussian/Poisson/Salt \& Pepper/Speckle Noise) to the given image to make it noisy one

3) Convert the given image to a row of length W2

4) Scan the image in blocks each of length 8

5) Apply DRT to the scanned vector and obtain the DRT spectral sequence

6) Preserve the 1st spectral component (CPI) \& midspectral component values and force other elements to zero

7) Repeat the same process until the entire image is scanned and processed

8) Resulting sequence is the sparsified spectral sequence

9) Apply Run Length Encoding (RLE) and compute the Compression Ratio

10) Apply decryption algorithm to obtain the spectral sequence

11) Apply inverse DRT on the spectral sequence to reconstruct the image which is noisy one

12) Remove noise from the reconstructed image using an appropriate filter to make it noise less one

13) Calculate Mean-Squared Error and Peak Signal-toNoise Ratio for this filtered reconstructed image with respect to the original input image.

Similar type of approach was carried out in the case of DCT and DWT. The results obtained are presented in the next section. 


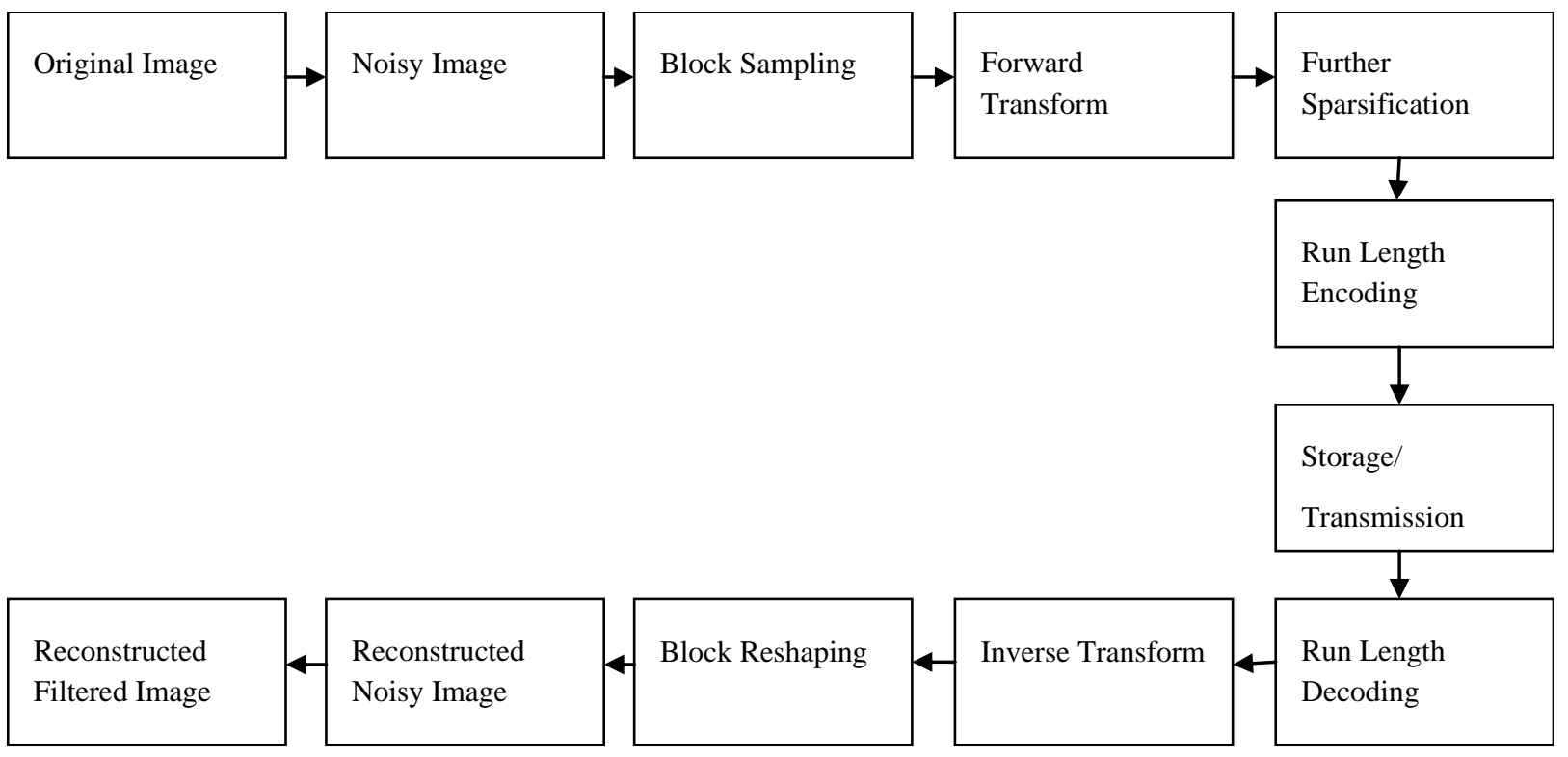

\section{SIMULATION RESULTS}

In order to have an in-depth analysis of the effect of noise on the performance of DRT and the other chosen candidate transforms, above algorithm has been considered. In this approach, the resulting PSNR and MSE have been compared for each candidate transform. By using this approach, the simulation Results are verified for four different types of noises, namely, Gaussian, Poisson, Salt \& Pepper and Speckle Noise. The simulation results obtained thus have been tabulated in Table 1. It can be observed that in the case of input image affected by Gaussian noise, Poisson noise, Salt \& pepper noise and speckle noise, DRT showed considerable improvement in PSNR compared to other candidate transforms. This can be extended further as there is a possibility of further improving the PSNR with the use of better noise filters. The images below depict the results discussed above:
In Figure 2 through Figure 5, column (a) describes the original images, column (b) describes the reconstructed images using discrete wavelet transform (DWT), column (c) describes the reconstructed images using discrete cosine transform (DCT), and column (d) describes the reconstructed images using discrete rajan transform (DRT). It is observed that the image reconstruction using DRT is better than that using other candidate transforms.

After simulation results it is evident that DRT method gives good and optimum results when compared to DWT and DCT in presence of four different and peculiar noises as per the perception of the quality of images. It is also clear that DRT method yields apt parametric results in terms PSNR, MSE and Compression Ratio. 
Table 1. Simulation Results

\begin{tabular}{|c|c|c|c|c|c|}
\hline $\begin{array}{l}\text { Type Of } \\
\text { Noise } \\
\text { Exists With } \\
\text { Image }\end{array}$ & Image & $\begin{array}{l}\text { Transform } \\
\text { Used }\end{array}$ & MSE & $\begin{array}{l}\text { PSNR(d } \\
\text { ecibels) }\end{array}$ & CR \\
\hline \multirow{9}{*}{$\begin{array}{l}\text { Gaussian } \\
\text { Noise }\end{array}$} & \multirow{3}{*}{ Lena Image } & DWT & $1.1061 \mathrm{e}+04$ & 7.6930 & 2.0064 \\
\hline & & DCT & 189.1790 & 25.3621 & 2.0049 \\
\hline & & DRT & 140.4490 & 26.6556 & 2.0042 \\
\hline & \multirow{3}{*}{ Boat Image } & DWT & $1.1742 \mathrm{e}+04$ & 7.4333 & 2.0059 \\
\hline & & DCT & 306.3658 & 23.2684 & 2.0045 \\
\hline & & DRT & 201.8582 & 25.0803 & 2.0039 \\
\hline & \multirow{3}{*}{ Zelda Image } & DWT & $6.2718 \mathrm{e}+03$ & 10.1569 & 2.0079 \\
\hline & & DCT & 124.7340 & 27.1710 & 2.0045 \\
\hline & & DRT & 110.1938 & 27.7092 & 2.0045 \\
\hline \multirow{9}{*}{$\begin{array}{l}\text { Poisson } \\
\text { Noise }\end{array}$} & \multirow{3}{*}{ Lena Image } & DWT & $1.1027 \mathrm{e}+04$ & 7.7062 & 2.0189 \\
\hline & & DCT & 131.4027 & 26.9448 & 2.0129 \\
\hline & & DRT & 67.5047 & 29.8375 & 2.0109 \\
\hline & \multirow{3}{*}{ Boat Image } & DWT & $1.1674 \mathrm{e}+04$ & 7.4586 & 1.0143 \\
\hline & & DCT & 240.3899 & 24.3216 & 2.0113 \\
\hline & & DRT & 120.6231 & 27.3165 & 2.0082 \\
\hline & \multirow{3}{*}{ Zelda Image } & DWT & $6.2438 \mathrm{e}+03$ & 10.1763 & 2.0241 \\
\hline & & DCT & 59.4413 & 30.3899 & 2.0160 \\
\hline & & DRT & 29.6878 & 33.4050 & 2.0112 \\
\hline \multirow{9}{*}{$\begin{array}{l}\text { Salt \& Pepper } \\
\text { Noise }\end{array}$} & \multirow{3}{*}{ Lena Image } & DWT & $1.3318 \mathrm{e}+04$ & 6.8864 & 2.0671 \\
\hline & & DCT & 129.2278 & 27.0172 & 2.0329 \\
\hline & & DRT & 61.2659 & 30.2586 & 2.0196 \\
\hline & \multirow{3}{*}{ Boat Image } & DWT & $1.4334 \mathrm{e}+04$ & 6.5671 & 2.0370 \\
\hline & & DCT & 256.5380 & 24.0393 & 2.0186 \\
\hline & & DRT & 132.7647 & 26.9000 & 2.0109 \\
\hline & \multirow{3}{*}{ Zelda Image } & DWT & $7.4894 \mathrm{e}+03$ & 9.3863 & 2.0620 \\
\hline & & DCT & 65.0723 & 29.9968 & 2.0087 \\
\hline & & DRT & 32.9990 & 32.9458 & 2.0152 \\
\hline \multirow{9}{*}{$\begin{array}{l}\text { Speckle } \\
\text { Noise }\end{array}$} & \multirow{3}{*}{ Lena Image } & DWT & $1.1104 \mathrm{e}+04$ & 7.6760 & 2.0095 \\
\hline & & DCT & 164.6373 & 25.9655 & 2.0075 \\
\hline & & DRT & 108.5385 & 27.7750 & 2.0063 \\
\hline & \multirow{3}{*}{ Boat Image } & DWT & $1.1754 \mathrm{e}+04$ & 7.4309 & 2.0087 \\
\hline & & DCT & 275.0982 & 23.7307 & 2.0064 \\
\hline & & DRT & 164.5720 & 25.9882 & 2.0054 \\
\hline & \multirow{3}{*}{ Zelda Image } & DWT & $6.2799 e+03$ & 10.1513 & 2.0136 \\
\hline & & DCT & 79.7642 & 29.1127 & 2.0100 \\
\hline & & DRT & 55.0099 & 30.7264 & 2.0074 \\
\hline
\end{tabular}



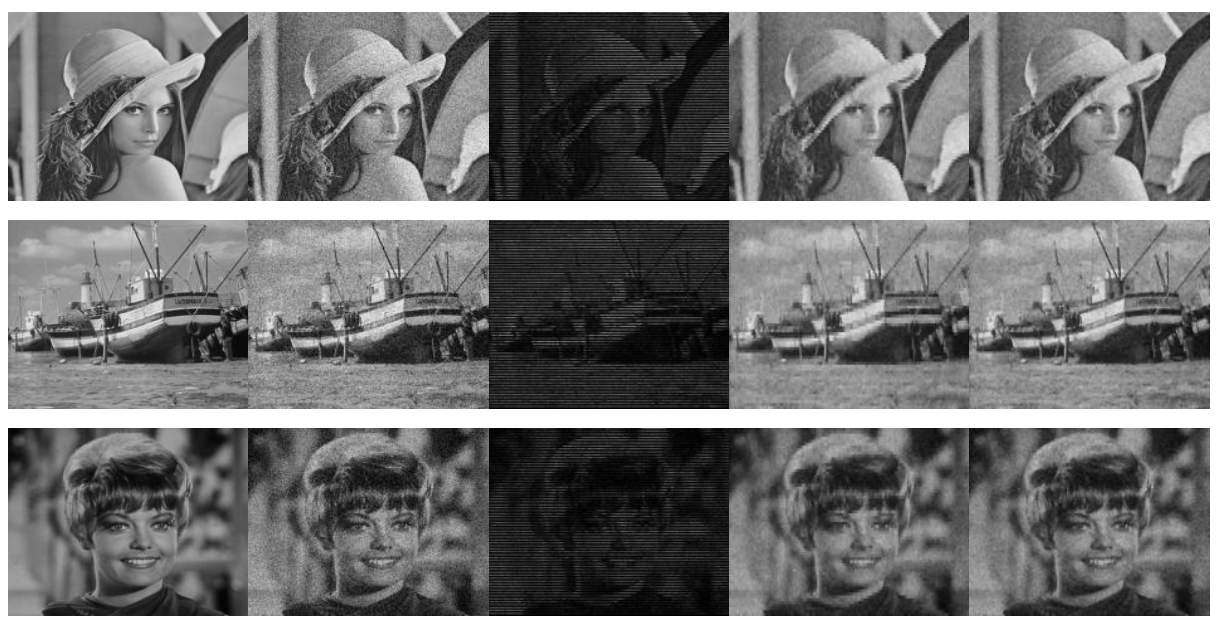

Fig 2: Simulation Results for Images with Gaussian Noise
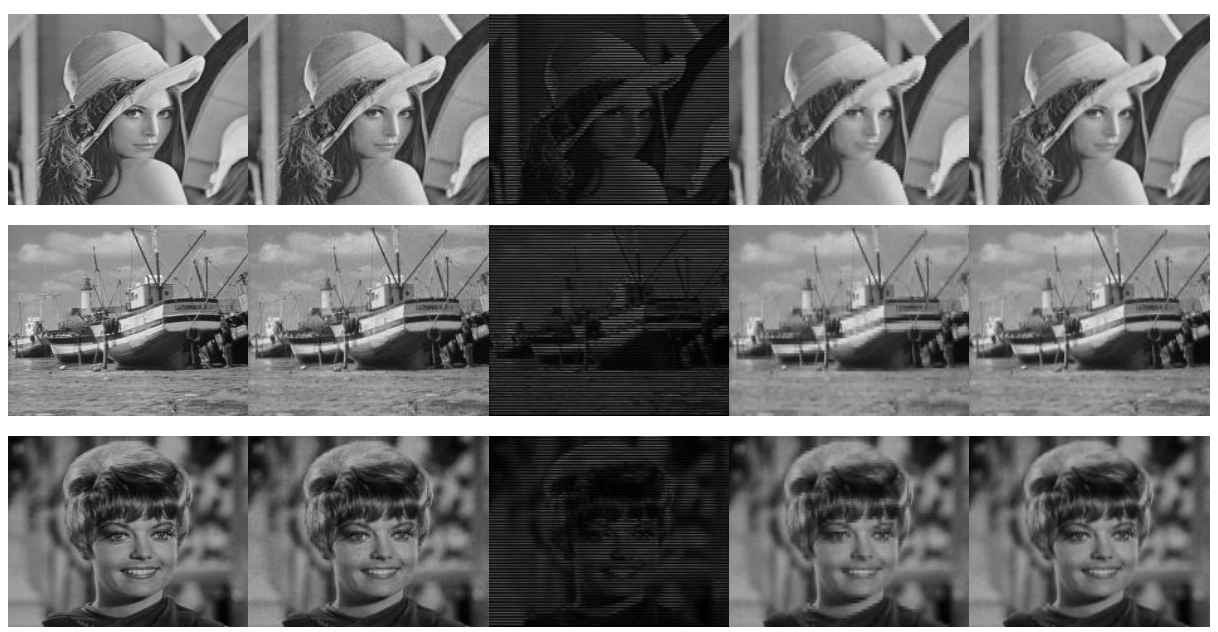

Fig 3: Simulation Results for Images with Poisson Noise
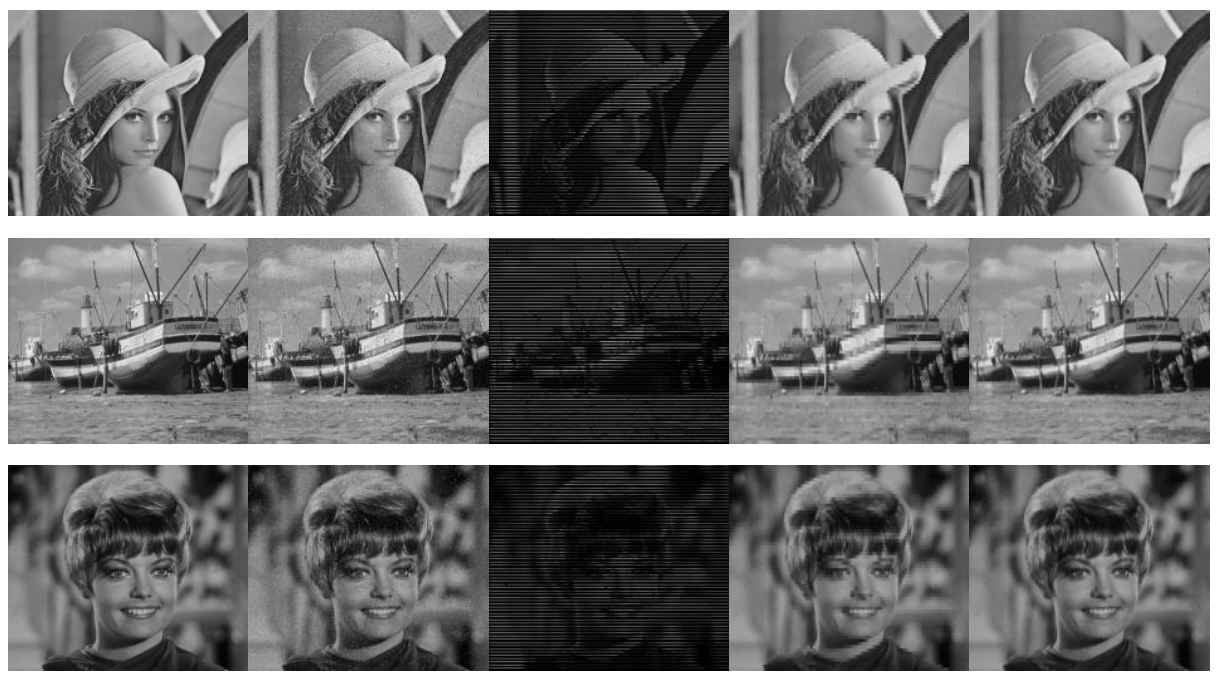

Fig 4: Simulation Results for Images with Salt \& Pepper Noise 

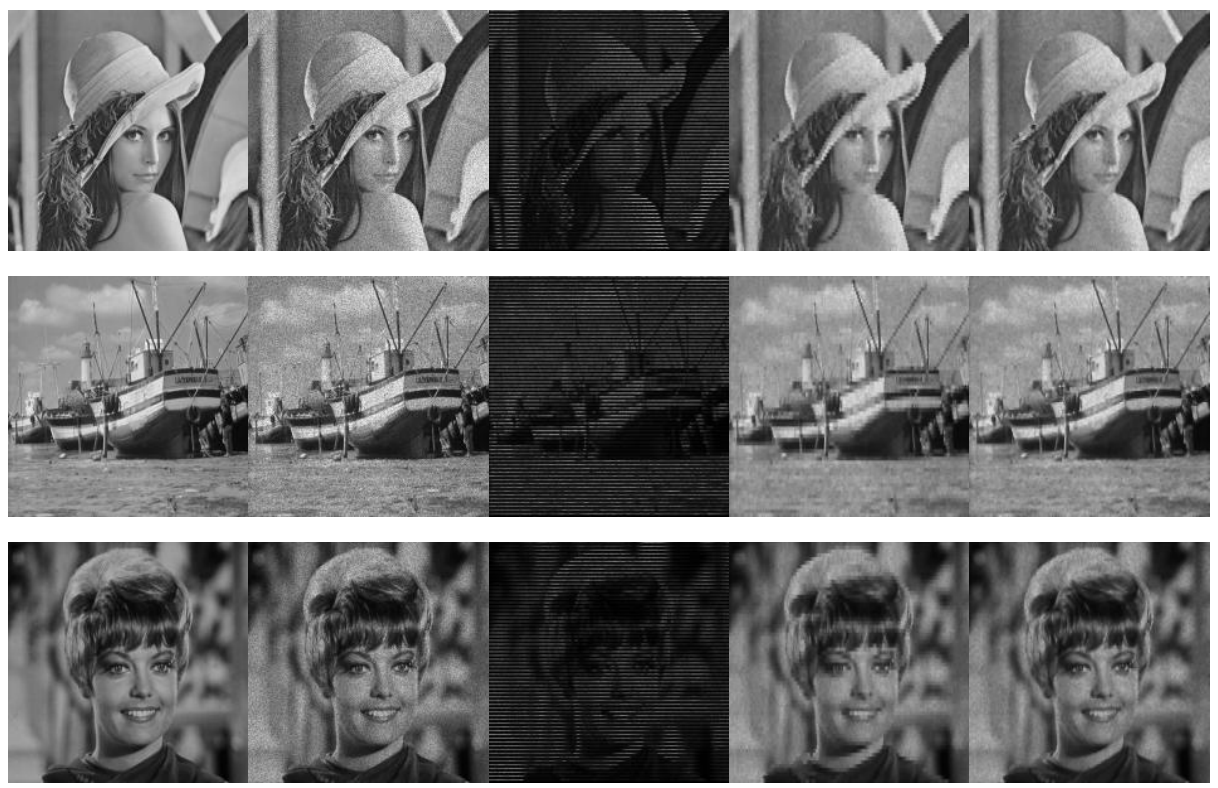

Fig 5: Simulation Results for Images with Speckle Noise

Column (i) Original Images,

Column (ii) Input Noisy Images, Column (iii) DWT Reconstructed Images, Column (iv) DCT Reconstructed Images, Column (v) DRT Reconstructed Images

\section{CONCLUSION}

As an option to these traditional approaches, the use of Discrete Rajan Transform (DRT) for sparsification and image compression was explored. Inverse Discrete Rajan Transform (IDRT) is used to retrieve back the input signal from its spectrum. Performance of DRT depends on the correlation value of a given data set, i.e., if the samples in the input data are spatially correlated (eg. image), then DRT shows better performance. In other words, performance of DRT is found to improve in a linear fashion with respect to the degree of correlation in input data. DRT yielded higher quality image than the other candidate transforms used. DRT is effective in introducing sparsity in images and thereby preserving the integrity of the images. Image Quality assessment plays an important role in various image processing applications. It is still an active area of research. A great deal of effort has been made in recent years to develop objective image quality metrics that correlate well with perceived human quality measurement or subjective methods. In this work Image quality assessment parameters have been derived to show that DRT exhibits optimum values.

\section{ACKNOWLEDGMENTS}

We are heartily thankful to Dr.E.G.Rajan, Director, Pentagram Research Centre, Hyderabad, India, and Ms.G.Prashanthi, MSc, Staffordshire University, UK, whose constant encouragement and guidance during the period of this work enabled us complete it with better perception of the subject.

\section{REFERENCES}

[1] Zhu Gui bin, Cao Chang xiu, Hu Zhong yu, He Shi biao, Sen Bai, "An image scrambling and encryption algorithm based on affine transformation [J]", Journal of Computer-Aided Design and Computer Graphics, Issue 6, pp. 711-715, 2003.
[2] En-hui Yang, Longji Wang, "Joint Optimization of RunLength Coding, Huffman Coding, and Quantization Table with Complete Baseline JPEG Decoder Compatibility [J]", IEEE Trans. Image Processing, Vol. 18, no. 1, pp.63-74, 2009.

[3] Q Xia, X Li, L Zhou, K M Lam, "Visual sensitivitybased low-bit-rate image compression algorithm[J]", IEEE Trans. Image Processing, Vol. 6, no. 7, pp. 910918, 2012.

[4] Zhiwei Xiong, Xiaoyan Su, Feng Wu, "Block-Based Image Compression with Parameter-Assistant Inpainting [J]", IEEE Trans. image processing, Vol. 19, no. 6, pp. 1651-1657, 2010.

[5] Xingsong Hou, Guizhong Liu, and Yiyang Zou, "SAR Image Data Compression Using Wavelet Packet Transform and Universal-Trellis Coded Quantization", IEEE Trans. Geoscience and Remote Sensing, Vol. 42, no. 11, pp. 2632-2641, November 2004.

[6] M. Zhang, G. Shao and K. Yi. "T-matrix and Its Applications in Image Processing [J]", Electronics Letters, Vol. 40, no. 25, pp. 1583-1584, 2004.

[7] Huibin Chang, K. Ng Michael, and Tieyong Zeng, "Reducing Artifacts in JPEG Decompression Via a Learned Dictionary", IEEE Trans. Signal Processing, vol. 62, no. 3, pp. 718-728 Feb. 2014.

[8] A. Skodras, C. Christopoulos, T. Ebrahimi, "The JPEG 2000 still-image compression standard", Signal Processing Magazine, IEEE, Vol. 18, no. 5, pp. 36-58, 2001.

[9] D. J. Jackson, S. J. Hannah, "Comparative analysis of image compression techniques", IEEE Conf. System Theory, 1993. Proceedings SSST '93, Twenty-Fifth Southeastern Symposium on, pp. 513-517, Mar. 1993. 
[10] Guangqi Shao, Yanping Wu, Yong A, Xiao Liu, Tiande Guo, "Fingerprint Compression Based on Sparse Representation", IEEE Trans. Image Processing, vol. 23, no. 2, pp. 489-501 Feb. 2014.

[11] Tanaya Guha, Rabab K. Ward, "Image Similarity Using Sparse Representation and Compression Distance", IEEE Trans. Multimedia, vol. 16, no. 4, pp. 980-987, June 2014.

[12] Jing-Ming Guo, Yun-Fu Liu, "Improved Block Truncation Coding Using Optimized Dot Diffusion", IEEE Trans. Image Processing, vol.23, no. 3, pp. 12691275, March 2014.

[13] Chuan Qin, Chin-Chen Chang, Yi-Ping Chiu, "A Novel Joint Data-Hiding and Compression Scheme Based on SMVQ and Image Inpainting", IEEE Trans. Image Processing, vol. 23, no. 3, pp. 969-978, March 2014.

[14] Shengli Chen, Xiaoxin Cheng, Jiapin Xu, "Research on image compression algorithm based on Rectangle Segmentation and storage with sparse matrix", 9th International Conf. Fuzzy Systems and Knowledge Discovery (FSKD), pp. 1904-1908, May 2012.

[15] Al-Shaykh, O.K.; Mersereau, R.M., "Lossy compression of noisy images", in Image Processing, IEEE Transactions on , vol.7, no.12, pp.1641-1652, Dec 1998.

[16] A. Macovski, Medical Imaging. Englewood Cliffs, NJ: Prentice-Hall, 1983.

[17] M. Rabbani, "Bayesian filtering of Poisson noise using local statistics", IEEE Trans. Acoust., Speech, Signal Processing, vol. 36, pp. 933-937, June 1988.

[18] W. Zeng, S. Daly, and S. Lei, "An overview of the visual optimization tools in JPEG 2000", Signal Processing: Image Communication, vol. 17, no. 1, pp. 85-104, 2002.

[19] D. M. Chandler, M. A. Masry, and S. S. Hemami, "Quantifying the visual quality of wavelet-compressed images based on local contrast, visual masking, and global precedence", in Proceedings of the 37th Asilomar Conference on Signals, Systems and Computers, pp. 1393-1397, November 2003.

[20] Z. Wang, A. C. Bovik, H. R. Sheikh, and E. P. Simoncelli, "Image quality assessment: from error visibility to structural similarity", IEEE Transactions on Image Processing, vol. 13, no.4, pp. 600-612, 2004.

[21] K. Egiazarian, J. Astola, N. Ponomarenko, V. Lukin, F. Battisti, and M. Carli, "New full-reference quality metrics based on HVS", in Proceedings of the 2nd International Workshop on Video Processing and Quality
Metrics, pp. 1-4, Scottsdale, Arizona, USA, 2006, CDROM.

[22] F. De Simone, D. Ticca, F. Dufaux, M. Ansorge, and T. Ebrahimi, "A comparative study of color image compression standards using perceptually driven quality metrics", in Applications of Digital Image Processing XXXI, vol. 7073 of Proceedings of SPIE, San Diego, Calif, USA, 2008.

[23] N. Ponomarenko, F. Silvestri, K. Egiazarian, J. Astola, M. Carli, and V. Lukin, "On between-coefficient contrast masking of DCT basis functions", in Proceedings of the 3rd International Workshop on Video Processing and Quality Metrics, pp. 1-4, Scottsdale, Ariz, USA, 2007, CD-ROM.

[24] G. K. Wallace, "The JPEG still picture compression standard", Communications of the ACM, vol. 34, no. 4, pp. 30-44, 1991.

[25] D. Taubman and M. Marcellin,JPEG 2000: Image Compression Fundamentals, Standards and Practice, Kluwer, Boston, Mass, USA, 2002.

[26] Li Zhiqianga, Sun Xiaoxin, Du Changbin, Ding Qun, "JPEG Algorithm Analysis and Application in Image Compression Encryption of Digital Chaos", IEEE Conf. , pp. 185-189, Sept. 2013.

[27] Elad Michael, "Sparse and Redundant Representations : From Theory to Applications in Signal and Image Processing", 2010, USA: Springer ISBN 978-1-44197011-4

[28] Ekambaram Naidu Mandalapu, Rajan E. G., 2009 , "Rajan Transform and its Uses in Pattern Recognition", Informatica 33, pp. 213-220.

[29] Kethepalli Mallikarjuna, Kodati Satya Prasad, and Makam Venkata Subramanyam, "Sparse Representation Based Image Compression Using Discrete Rajan Transform", International Journal of Applied Engineering Research (IJAER), Vol. 10, No. 13, 2015 , pp.33424-33429.

[30] Govindarajan Prashanthi, "Signal Sparsification with Discrete Rajan Transform (DRT): Principles, Properties and Applications", MSc, Staffordshire University, UK, 2012.

[31] Kethepalli Mallikarjuna, K.Satya Prasad, and M.V.Subramanyam, "Sparsification of Digital Images using Discrete Rajan Transform", Journal of Information Processing Systems(JIPS), 2015, in press. 